\title{
Discrete Lattice Systems and the Equivalence of Microcanonical, Canonical and Grand Canonical Gibbs States
}

\author{
Paul Vanheuverzwijn $\star$ \\ Instituut voor Theoretische Fysica, Universiteit Leuven, B-3030 Leuven, Belgium
}

\begin{abstract}
It is proven that a microcanonical Gibbs measure on a classical discrete lattice system is a mixture of canonical Gibbs measures, provided the potential is "approximately periodic," has finite range and possesses a commensurability property. No periodicity is imposed on the measure. When the potential is not approximately periodic or does not have the commensurability property, the inclusion does not hold.

As a by-product, a new proof is given of the fact that for a large class of potentials, a canonical Gibbs measure is a mixture of grand canonical measures. Thus the equivalence of ensembles is obtained in the sense of identical correlation functions.
\end{abstract}

\section{Introduction}

There is a long standing tradition in statistical mechanics of modelling in terms of classical discrete lattice systems: lattice gases and spin systems (including the Ising, Ashkin-Teller, Potts and $Z_{n}$ models) are amongst the most intensively studied objects in this research area [1]. A general and rigorous theory of their equilibrium properties and phase transitions was recently undertaken by Pirogov and Sinai $[2,3]$ and continued in [4] for instance. The mathematical context is that of a stochastic process taking values in some finite set, and indexed by the finite subsets of an infinite lattice. The underlying measure is a grand canonical Gibbs measure defined on the configuration space for the infinite system.

The present article is an attempt to justify this ansatz. Indeed, there are more natural choices for the equilibrium measure: the canonical and microcanonical Gibbs measures, for instance. We shall, however, prove the equivalence of the three descriptions in the context of infinite systems. On a thermodynamical level, the equivalence is well established in that Legendre transformations were shown to hold 
between the thermodynamical functions. But whether the three ensembles lead to identical correlation functions, i.e. whether the measures are identical, is a much more involved problem, not in the least by the occurrence of inhomogeneous measures and interactions. It is the latter problem which is the main concern of this paper.

The DLR equations [5, 6] or suitable adaptations of these to the canonical [7] or microcanonical [8-10] setting, rigorously define the equilibrium. (See definitions $2.2,2.3,2.4$ ). Letting $\mathscr{G}(\beta, z)$ denote the set of grand canonical Gibbs measures (or states) at an inverse temperature $\beta$ and a chemical potential (vector) $z, \mathscr{C}(\beta)$ the set of canonical Gibbs states at an inverse temperature $\beta$ and $\mathscr{M}$ the set of microcanonical Gibbs states (all in connection with a fixed potential), the main problems to be solved are the questions when $\mathscr{M} \subset \bigcup_{\beta} \mathscr{C}(\beta)$ and when in turn $\mathscr{C}(\beta) \subset \bigcup_{z} \mathscr{G}(\beta, z)$, (the reverse inclusions holding in complete generality). In particular, the second inclusion has been the object of numerous investigations, culminating in Georgii's work [7]. For a wide class of potentials, including some non-uniformly bounded inhomogeneous cases, it was shown that an extreme element of $\mathscr{C}(\beta)$, is an extreme element of $\mathscr{G}(\beta, z)$ for some $z$, thus solving the problem, since $\mathscr{C}(\beta)$ and $\mathscr{G}(\beta, z)$ are Choquet simplices. When the potential is uniformly bounded, there is no further assumption involved [7, Theorem 5.15] and in general, it is conjectured that the equivalence holds as soon as with non-zero probability there is one particle of every type (in the lattice gas language). An inhomogeneous, not uniformly bounded model, supporting this conjecture was studied in [11].

Much less is known as to the validity of the inclusion $\mathscr{M} \subset \bigcup_{\beta} \mathscr{C}(\beta)$ (or the corresponding one for the extreme elements). Under the extra hypothesis of translation invariance of the measures and an additional technical assumption (a sufficient condition for its validity being that all local probabilities be strictly positive). Thompson proved the statement for translation invariant, finite range potentials with a certain commensurability property. [8, Theorem 3.1. and Lemma 3.7].

Denoting by ex $\mathscr{M}, \operatorname{ex} \mathscr{C}(\beta), \ldots$ the set of extreme elements in $\mathscr{M}$ etc. our main result (Theorems 3.3 and 3.4) shows that the inclusion ex $\mathscr{M} \subset \bigcup_{\beta} \operatorname{ex} \mathscr{C}(\beta)$ holds without the assumption of translation invariance of the measures nor of the interaction, but it is assumed that no local event has zero probability. This hypothesis is not restrictive if we wish to obtain elements in $\mathscr{C}(\beta)$ with finite $\beta$, or states in $\mathscr{G}(\beta, z)$ with finite $\beta$ and nonzero $z$. Our conditions on the potential are: "approximate periodicity" (Definition 2.1), finite range and a certain commensurability property. The first hypothesis is weak enough so as to include disordered systems and roughly means that every local energy value should occur infinitely often. (We allow for multiple component spins, and many-body interactions.) We expect that microcanonical Gibbs measures with vanishing local probabilities are either ground states or ceiling states, thus belonging to $\mathscr{C}(\infty)$ or $\mathscr{C}(-\infty)$ respectively, or else have the property of excluding certain particle types. In Remark 3.8, a few examples are given.

Analogous results have been obtained before, for continuous systems by Aizenman, Goldstein and Lebowitz [9], and in a more abstract setting by Preston in 
[10]. The discreteness of the spectrum and the non-periodicity require different techniques. It should be possible however, to transpose the basic idea, a straightforward application of the microcanonical hypothesis and of cluster properties, to continuous systems. In fact, this basic step is a development of an idea we used before, in collaboration with W. Sullivan, $([11])$ to prove the equivalence of the canonical and grand canonical ensembles in a certain highly inhomogeneous Ising model. For the benefit of the reader we have included, in Theorem 3.5., an adaptation of this proof to the equivalence canonical-grandcanonical in model systems with uniformly bounded potentials. This proof may serve as an introduction to the proof of the equivalence canonical-microcanonical which is more involved and which we describe here.

Let $\mu$ be an extreme microcanonical Gibbs state. The microcanonical DLR equations (see Definition 2.4 below) prescribe equal probabilities to all local configurations in a certain finite volume, having the same particle numbers and the same conditional energy for a given boundary condition. Assume that two local configurations $\xi$ and $\xi^{\prime}$ are given in some volume $\Lambda$, with equal particle numbers but not necessarily equal energy. We may assume in addition that $\xi$ and $\xi^{\prime}$ are equal on the "inner boundary $\delta \Lambda . "$ ( $\delta \Lambda$ denotes the set of sites in $\Lambda$, interacting with $\Lambda^{c}$.) Assume that the potential is periodic; it is then possible to find a volume $\Delta$, a large distance away from $\Lambda$, and two configurations $\eta$ and $\eta^{\prime}$ in $\Delta$ such that the joint configurations $(\xi, \eta)$ and $\left(\xi^{\prime}, \eta^{\prime}\right)$ in $\Lambda \cup \Delta$ have the same particle numbers, the same energy value and are equal on $\delta(\Lambda \cup \Delta)$. Therefore, by the microcanonical hypothesis, $(\xi, \eta)$ and $\left(\xi^{\prime}, \eta^{\prime}\right)$ have equal probabilities: $\mu_{\Lambda \cup \Delta}(\xi, \eta)=\mu_{\Lambda \cup \Lambda}\left(\xi^{\prime}, \eta^{\prime}\right)$. Since $\mu$ is extreme, it is clustering and we have $\mu_{\Lambda}(\xi) \mu_{\Delta}(\eta) \simeq \mu_{\Lambda \cup \Delta}(\xi, \eta)=$ $\mu_{\Lambda \cup \Delta}\left(\xi^{\prime}, \eta^{\prime}\right) \simeq \mu_{\Lambda}\left(\xi^{\prime}\right) \mu_{\Delta}\left(\eta^{\prime}\right)$. By construction, we have in addition that the particle numbers of $\eta$ and $\eta^{\prime}$ are equal and that the energy difference between $\xi$ and $\xi^{\prime}$ equals that between $\eta^{\prime}$ and $\eta$. In the limit where the distance $(\Lambda, \Delta)$ tends to infinity we obtain, provided all local events have strictly positive probabilities, that

$$
\mu_{\Lambda}(\xi) / \mu_{\Lambda}\left(\xi^{\prime}\right)=\mu_{\Delta}\left(\eta^{\prime}\right) / \mu_{\Delta}(\eta)
$$

and it is not hard to see that (1.4) implies the existence of a function $f$ of exponential type of the energy difference such that $\mu_{\Lambda}(\xi) / \mu_{\Lambda}\left(\xi^{\prime}\right)=f\left(E(\xi)-E\left(\xi^{\prime}\right)\right)$. In view of the relation between $\xi$ and $\xi^{\prime}$, and in view of the commensurability condition, this implies the canonical DLR equations: local configurations with the same particle numbers have probabilities whose quotient equals the quotient of the exponentials of their energy values. (allowing for negative temperatures).

When the potential does not have the commensurability property or when it is not approximately periodic, it is not hard to find examples of microcanonical Gibbs measures that are not canonical (Example 3.7 and Remark 3.9). On the other hand, the condition that no local configuration has zero probability is necessary in order to obtain canonical Gibbs measures at finite inverse temperatures. It is well known that grand canonical (g.c.) and canonical Gibbs measures at finite temperatures, and admitting all particle types, have strictly positive probabilities for all local events. (In the g.c. case, this prevails when no $z$-component vanishes.)

To further clarify the condition that there be no local events having probability zero (i.e. that the measure be everywhere dense), we shall study elsewhere the one 
dimensional chain with translation invariant interactions in more detail. In complete analogy with the property that with non-zero probability there is a particle of every type (in the canonical case this implies an infinity of particles of every type), we show that a microcanonical Gibbs measure in one dimensional systems, is everywhere dense, as soon as with non-zero probability there are infinitely many basic configurations of every type. In a chain with nearest neighbour interactions at most, a basic configuration is a configuration in a set consisting of two nearest neighbour sites. Hence in a $(+1,-1)$ nearest neighbour chain, there are four basic configurations: $(+1,+1),(+1,-1),(-1,+1)$ and $(+1,+1)$, and these events should occur infinitely often in order for the microcanonical measure to be everywhere dense.

In general, if the potential is of range $D$, the chain is partitioned in intervals (cells) of length $(D-1)$, and a basic configuration is a configuration in a set consisting of two nearest neighbour cells.

In summary, we have obtained a clear understanding of when an extreme microcanonical Gibbs measure $\mu$ is a canonical Gibbs measure for some finite inverse temperature $\beta$. It is necessary and sufficient that $\mu$ be everywhere dense, a property which for one-dimensional systems is equivalent to the occurrence of infinitely many basic configurations of every type. We conjecture that not everywhere dense microcanonical Gibbs states are precisely the ground or ceiling states, or else, have the property of excluding certain particle types. Additional open problems are listed in Sect 5 .

The reader interested in the main results only is referred to Theorems 3.3 and 3.4, Examples 3.7, 3.8 and 3.9 and the proofs in Sect. 4.

\section{Microcanonical Gibbs States}

Introduce a finite set $F$ whose elements $a, b, \ldots$ represent the different types of particles (or spin values). The particles are supposed to live on a lattice $L$, which for simplicity will be taken to be $\mathbb{Z}^{v}$; if multiple occupation of lattice sites is excluded, the configuration space for the infinite system is $\Omega \equiv F^{L}$. For $\Lambda \subset L$, let $\Omega_{\Lambda}=F^{\Lambda}$. Configurations in $\Omega$ are denoted by $\xi, \eta, \ldots$; their projections onto $\Omega_{\Lambda}$ ("local configurations") by $\xi_{A}, \eta_{\Lambda}, \cdots$ and when $\Lambda=\{x\}, x \in L, \xi_{\{x\}}$ will be written $\xi_{x}$. If no confusion can arise, the reference to $\Lambda$ will be omitted. If $\Lambda_{1} \cap \Lambda_{2}=\phi$ and $\xi \in \Omega_{\Lambda_{1}}$, $\eta \in \Omega_{\Lambda_{2}}$, then $(\xi, \eta)$ or $\xi, \eta$ or $\xi \eta$ denote the joint configuration in $\Lambda_{1} \cup \Lambda_{2}$.

The symbol $I_{\omega_{\Lambda}}$ (or $I_{\omega}$ ) denotes the characteristic function for the event $\left\{\xi: \xi_{\Delta}=\omega_{A}\right\}$.

Often it is convenient to reformulate the model as a stochastic process taking values in $F$ and parametrized by the set of sites in $L$. Random variables $S_{x}(x \in L)$ and $S_{\Lambda}(\Lambda \subset L)$ are defined by $S_{x}(\xi)=\xi_{x}, S_{\Lambda}(\xi)=\xi_{\Lambda}$.

For every point a in $F$, (particle) number variables $N_{A}^{a}$ are defined by $N_{\Delta}^{a}(\xi)=\left|\left\{x \in \Lambda \mid \xi_{x}=a\right\}\right|$. Let $N^{a}=N_{L}^{a}$. Here and in the following the symbol $|$.$| denotes the cardinality of the involved set. Finally the following$ vector valued number variables will be used: $N_{\Lambda}(\xi)=\left(N_{\Lambda}^{a}(\xi)\right)_{a \in F}$ and $N(\xi)=N_{L}(\xi)$. In this connection, we denote by $I_{\Lambda}^{N_{\Lambda}(\eta)}($.$) the characteristic function for the event$ $N_{\Lambda}=N_{\Lambda}(\eta)$. 
By a potential $\Phi$ we mean a family $\left\{\phi_{\Delta}\right\}$ of real valued functions on $\Omega$, indexed by the finite sets in $L$, such that $\phi_{\Delta}(\xi)$ depends on $\xi_{\Delta}$ only. By definition $\phi_{\Delta}(\xi)=\phi_{\Delta}\left(\xi_{\Delta}\right)$.

Throughout the paper, the potential is assumed to have a finite range $D$ and to be "approximately periodic" as will be explained shortly. For some of the final results, the following commensurability condition must be imposed:

$$
\forall \xi, \forall \eta, \forall \Delta_{1}, \forall \Delta_{2}: \phi_{\Delta_{1}}(\xi) / \phi_{\Delta_{2}}(\eta) \in \mathbb{Q}
$$

whenever $\phi_{\Delta_{2}}(\eta) \neq 0$.

With respect to a finite range potential $\Phi$, it is useful to introduce for a finite set $\Lambda$ in $L$ :

$$
\begin{aligned}
\partial \Lambda & =\left\{x \in \Lambda^{c}: \operatorname{dist}(x, \Lambda) \leqq D\right\}, \\
\delta \Lambda & =\left\{x \in \Lambda: \operatorname{dist}\left(x, \Lambda^{c}\right) \leqq D\right\}, \\
\Lambda & =\Lambda \cup \partial \Lambda .
\end{aligned}
$$

The internal energy $E_{\Lambda}(\xi)$ for $\xi \in \Omega_{\Lambda}, \Lambda$ finite is defined by:

$$
E_{\Lambda}(\xi)=\sum_{\Delta \subset \Lambda} \phi_{\Delta}(\xi)
$$

and for a given boundary condition $\eta$ in $\Omega_{\Lambda^{c}}$, the conditional energy of $\xi$ in $\Omega_{\Lambda}$ is defined by

$$
E_{\Lambda}(\xi \mid \eta)=\sum_{\Delta ; \Delta \cap \Lambda \neq \phi} \phi_{\Delta}(\xi, \eta)
$$

Equation (2.2) in turn defines the following function:

$I_{\Lambda}^{E_{A}\left(\eta_{A} \mid \eta^{c}\right)}($.$) is the characteristic function for the event E_{\Lambda}\left(\cdot \mid \eta_{\Lambda^{c}}\right)=E_{\Lambda}\left(\eta_{\Lambda} \mid \eta_{\Lambda^{c}}\right)$.

For $i \in L$, let $T_{i}$ be the translation defined by

$$
\begin{aligned}
& \forall \Lambda \subset L: T_{i}(\Lambda)=\Lambda+i \\
& \forall \xi \in \Omega:\left[T_{i}(\xi)\right]_{k}=\xi_{k-i} \quad \forall k \in L .
\end{aligned}
$$

Occasionally, an arbitrary translation will be denoted by $T$.

Definition 2.1. A (finite range) potential $\Phi$ is said to be approximately periodic if for all finite $\Lambda$ there exists an adapted sequence $\left\{\Lambda_{n}\right\}_{\pi}$ with respect to $\Phi$, i.e.:

There exist $k_{n} \in L$ such that

$$
\begin{aligned}
& \Lambda_{n}=T_{k_{n}}(\Lambda), \\
& \operatorname{dist}\left(\Lambda, \Lambda_{n}\right) \rightarrow \infty \text { as } n \rightarrow \infty, \\
& E_{\Lambda}(. \mid .)=E_{\Lambda_{n}}\left(T_{k_{n}}(.) \mid T_{k_{n}}(.)\right) .
\end{aligned}
$$

Loosely speaking, every local energy value should occur infinitely often in the lattice. Clearly, translation invariant or periodic potentials (i.e. potentials that are invariant under some subgroup of $L$ ) are approximately periodic, but it appears that the notion comprises in addition the potentials that typically occur in disordered systems. See Remark A.1 in the appendix.

For $\alpha$ in some $\Omega_{\Delta}$, let $N_{\Lambda}^{\alpha}(\xi)$ denote

$$
N_{\Lambda}^{\alpha}(\xi)=\mid\left\{n \in L \mid T_{n}(\Delta)=\Delta_{n} \text { with } \Delta_{n} \subset \Lambda \text { and } T_{n}(\alpha)=\xi_{\Delta_{n}}\right\} \mid .
$$


When the potential is translation invariant, it is possible to find coupling constants $J_{\alpha}$, indexed by the configurations in some $\mathscr{A} \subset \Omega_{\Delta}$ ( $\Delta$ centred around the origin), such that

$$
E_{\Lambda}(\xi \mid \eta)=\sum_{\alpha \in \mathscr{A}} J_{\alpha}\left[N_{\bar{\lambda}}^{\alpha}(\xi, \eta)-N_{\partial \Lambda}^{\alpha}(\xi, \eta)\right] .
$$

It is assumed that the set $\mathscr{A}$ is chosen to be minimal. The commensurability hypothesis then reduces to: $J_{\alpha} / J_{\beta} \in \mathbb{Q} \forall \alpha, \beta$ in $\mathscr{A}$ with $J_{\beta} \neq 0$.

As an example, the nearest neighbour Ising chain (with $F=\{1,-1\}$ )

$$
E_{\Lambda}(\xi \mid \eta)=-J \sum_{\mathrm{i} ;[, 1,+1] \cap \Lambda \neq \phi}\left(S_{i} S_{i+1}\right)(\xi, \eta)
$$

may be written as in (2.3) using the following objects: $\Delta=[0,1] ; \mathscr{A}=\{\alpha=(1,1)$, $\beta=(-1,-1), \gamma=(1,-1), \delta=(-1,1)\} \subset \Omega_{\Delta}$ and $J_{\alpha}=J_{\beta}=-J ; J_{\gamma}=J_{\delta}=+J$.

The following $\sigma$-algebras are essential for the definition of Gibbs states. (An algebraic formulation is given in the appendix, Remark A.2.) $\mathscr{F}$ is the algebra generated by the product topology on $\Omega$ (with the discrete topology on $F$ ). $\mathscr{F}_{A}$ is the algebra generated by $\left\{S_{x}, x \in \Lambda^{c}\right\}, \mathscr{E}_{\Lambda}$ is generated by $\mathscr{F}_{\Lambda}$ and $N_{\Lambda}$, and $\mathscr{D}_{A}$ is the algebra generated by $\mathscr{E}_{\Lambda}$ and $E_{\Lambda}(. \mid$.$) . The tail field \mathscr{F}_{\infty}=\cap \mathscr{F}_{\Lambda}$, the symmetric field $\mathscr{E}_{\infty}=\cap \mathscr{E}_{A}$ and the field $\mathscr{D}_{\infty}=\cap \mathscr{D}_{A}$ are defined by intersection over all finite sets in $L$. If $\mu$ is a probability measure on $(\Omega, \mathscr{F}), \mu_{\Lambda}\left(. \mid \mathscr{F}_{\Lambda}\right)$ denotes the conditional probability of an event in $\Lambda$ with respect to $\mu$ and $\mathscr{F}_{\Lambda} \cdot \mu_{\Lambda}\left(. \mid \mathscr{E}_{A}\right)$ and $\mu_{\Lambda}\left(. \mid \mathscr{D}_{\Lambda}\right)$ have analogous interpretations.

Definition 2.2 [5-7]. A (regular Borel) probability measure $\mu$ on $(\Omega, \mathscr{F})$ is a grand canonical Gibbs measure (or state) for the potential $\Phi$, at an inverse temperature $\beta$ and an activity $z\left(\right.$ a vector in $\mathbb{R}_{+}^{F}$ with $\left.\sum_{a \in F} z(a)>0\right)$ if for all finite $\Lambda$ in $L$, and all $\xi$ in $\Omega_{\Lambda}$ :

$$
\mu_{\Lambda}\left(\xi \mid \mathscr{F}_{\Lambda}\right) \equiv \mu_{\Lambda}\left(S_{\Lambda}=\xi \mid \mathscr{F}_{\Lambda}\right)=\gamma_{\Lambda}^{z}(\xi \mid .) \mu \text { a.s. }
$$

with

where

$$
\gamma_{\Lambda}^{z}(\xi \mid \eta)=\left[Z_{\Lambda, z}(\eta)\right]^{-1} \cdot \prod_{a \in F}[z(a)]^{N_{\Lambda}^{a}(\xi)} \cdot \exp \left[-\beta E_{\Lambda}(\xi \mid \eta)\right]
$$

$$
Z_{\Lambda, z}(\eta) \text { is a normalization factor. }
$$

Definition 2.3 [7]. A (regular Borel) probability measure $\mu$ on $(\Omega, \mathscr{F})$ is a canonical Gibbs measure for $\Phi$, at an inverse temperature $\beta$ iff for all finite $\Lambda$, and all $\xi$ in $\Omega_{\Lambda}$ :

$$
\mu_{\Lambda}\left(\xi \mid \mathscr{E}_{\Lambda}\right)=\gamma_{\Lambda, N_{A^{\prime}}(.)}(\xi \mid .) \quad \mu \text { a.s. }
$$

with

$$
\gamma_{\Lambda, N_{\Lambda}(\eta)}(\xi \mid \eta)=I_{\Lambda}^{N_{\Lambda}(\eta)}(\xi) \cdot \exp \left[-\beta E_{\Lambda}(\xi \mid \eta)\right] \cdot\left[Z_{\Lambda, N_{\Lambda}(\eta)}(\eta)^{-1}\right.
$$

where $Z_{\Lambda, N_{A}(\eta)}(\eta)$ is a normalization factor.

Definition 2.4 [8-10]. A (regular Borel) probability measure $\mu$ on $(\Omega, \mathscr{F})$ is a micro- 
canonical Gibbs measure for $\Phi$ iff for all finite $\Lambda$ and all $\xi$ in $\Omega_{\Lambda}$ :

$$
\mu_{\Lambda}\left(\xi \mid \mathscr{D}_{\Lambda}\right)=v_{\Lambda, N_{\Lambda}(), E_{A}(\mid)}(\xi \mid .) \quad \mu \text { a.s., }
$$

with

$$
\begin{aligned}
& v_{A, N_{A}(\eta), E_{A}\left(\eta_{A} \mid \eta_{A}^{c}\right)}(\xi \mid \eta)=I_{\Lambda}^{N_{\Lambda}(\eta)}(\xi) I_{\Lambda}^{E_{\Lambda}\left(\eta_{A} \mid \eta_{A}^{c}\right)}(\xi)\left[Z_{A, N_{\Lambda}(\eta), E_{A}\left(\eta_{A} \mid \eta_{A}^{c}\right)}\right]^{-1} \\
& \equiv v_{\Lambda}(\xi \mid \eta) \text {. }
\end{aligned}
$$

The set $\mathscr{M}$ of all microcanonical Gibbs states is nonempty: ground states belong to $\mathscr{M}$. More generally: whenever a sequence of microcanonical Gibbs distributions $\left\{v_{\Lambda}\left(. \mid \eta_{A^{c}}\right)\right\}_{\Lambda}$ converges - and by compactness every sequence has a convergent subsequence-its limit is a microcanonical Gibbs state.

In a standard way, one shows that the set of microcanonical Gibbs measures is a Choquet simplex, and that a microcanonical Gibbs state is extreme in that set iff $\mathscr{D}_{\infty}$ is trivial. (It suffices to show that if $\mu($.$) and \mu(f$.) are both microcanonical Gibbs states, then $f \in \mathscr{D}_{\infty}$ and conversely.) (Compare [12, Theorem 2.1], [8, Lemma 3.7], or see Remark A2 in the appendix.)

As a consequence it is sufficient to study extreme microcanonical Gibbs states.

Let us remark that the microcanonical hypothesis will enter only through a simplified statement as described in Lemma 4.1.

\section{The Main Results}

In the present context, a measure $\mu$ is everywhere dense (that is, $\mu(\theta)>0$ for every nonempty open subset $\theta$ of $\Omega$ ) iff for all finite $\Lambda$ and all $\xi$ in $\Omega_{\Lambda}, \mu_{\Lambda}(\xi) \equiv \mu\left(S_{\Lambda}=\xi\right)>0$.

The main results - holding for everywhere dense measures - will be formulated in this section. Most proofs will be given in Sect. 4, and the notion of everywhere dense microcanonical Gibbs measure will be clarified in Sect. 5 .

Definition 3.1. The spectrum $\Sigma(\Phi)$ (or $\Sigma$ if no confusion can arise) of a finite range potential $\Phi$ is defined to be the set of all differences of the form $(\sigma-\tau)$ with $\sigma=E_{\Lambda}(\omega), \tau=E_{\Lambda}(\eta)$, with $\omega, \eta$ belonging to some finite $\Omega_{\Lambda}$, with $N_{\Lambda}(\omega)=N_{\Lambda}(\eta)$ and $\omega_{\delta \Lambda}=\eta_{\delta \Lambda}$.

Remark 3.2. If $\Phi$ is a linear combination of the number variables $N^{a}$, that is, $E_{\Lambda}(\xi \mid \eta)=E_{\Lambda}(\xi)=\sum_{a \in F} J_{a} N_{\Lambda}^{a}(\xi)$, for some family of $J_{a}$, then clearly, $\Sigma(\Phi)=\{0\}$.

The converse statement, however is not necessarily true. The following is a counterexample.

Let $v=1$ and $F=\{a, b\}$, and in the notation of (2.3), let

$$
E_{\Lambda}(\xi \mid \eta)=\sum_{x, y \in F} J_{x y}\left[N_{\Lambda}^{x y}(\xi, \eta)-N_{\partial \Lambda}^{x y}(\xi, \eta)\right]
$$

with $N_{\Lambda}^{x y}(\xi ; \eta)$ denoting the number of couples of nearest neighbour sites in $\Lambda$ of the form $x y$, contained in $(\xi, \eta)$. Thus $D=1$, but it follows that $\Sigma(\Phi)=\{0\}$ iff $J_{a a}+$ $J_{b b}=J_{a b}+J_{b a}$. The proof will be omitted.

However, if-again for general $F-F$ contains a point 0 (to be interpreted as a 
vacancy) with the property that there is a normalization of the energy such that $\forall \Lambda$, $\forall \omega$ in $\Omega_{\Lambda}, \forall k \notin \Lambda$ :

$$
E_{\Lambda}(\omega)=E_{\Lambda \cup\{k\}}(\omega, 0),
$$

then indeed $\Sigma(\Phi)=\{0\}$ iff $\Phi$ is linearly dependent on the particle number variables. Again the proof will be omitted.

The main result now reads:

Theorem 3.3. If $\mu$ is an everywhere dense, extreme microcanonical Gibbs measure for an approximately periodic, finite range potential $\Phi$ with commensurable coupling constants, then $\mu$ is an extreme canonical Gibbs measure for some finite (but not necessarily positive) inverse temperature $\beta$. Moreover $\mu\left(N^{a}=\infty \forall a \in F\right)>0$ and $\beta$ is unique iff $\Sigma(\Phi) \neq\{0\}$.

Conversely, if $\mu$ is a canonical Gibbs measure with $\mu\left(N^{a} \geqq 1 \forall a \in F\right)>0$, then $\mu$ is an everywhere dense microcanonical Gibbs measure. (Approximate periodicity or commensurability are not needed for this implication to hold.)

Theorem 3.4. If in addition to the hypotheses in the first part of Theorem 3.3, there exists a finite $\Lambda$, and $\xi, \eta$ in $\Omega_{\Lambda}$ with

i) $\xi_{\delta \Lambda}=\eta_{\delta \Lambda}$,

ii) $N_{\Lambda}(\xi)=N_{\Lambda}(\eta)$

iii) $E_{\Lambda}(\xi)<E_{\Lambda}(\eta)$

and iv) $\mu_{\Lambda}(\xi)>\mu_{\Lambda}(\eta)$,

then $\mu$ is a canonical Gibbs measure for some positive $\beta$.

The proof of Theorem 3.3 will be postponed to Sect. 4 . Theorem 3.4 on the other hand, is an easy consequence of Theorem 3.3.

The next result does not hinge on the approximate periodicity nor on the commensurability of the potential, but holds for the class of uniformly bounded potentials, that is $\Phi$ satisfies

$$
\sup _{x \in L} \sum_{\Lambda \ni x}\left\|\phi_{\Lambda}\right\|_{\infty}<\infty
$$

Although the equivalence canonical-grandcanonical was prove in much more generality by Georgii [7, Theorem 5.15], we include a proof as it may help to clarify the basic idea in the more difficult case of the equivalence microcanonicalcanonical. The proof is basically that of Theorem 3.2 in [11].

Theorem 3.5. If $\mu$ is an extreme canonical Gibbs measure for some finite inverse temperature $\beta$ and some uniformly bounded finite range potential $\Phi$, then $\mu$ is an extreme grand canonical Gibbs measure for $\beta$ and some activity vector $z$, with $z_{a}(a \in F) \neq 0$ whenever with non-zero probability there is a particle of type a. Conversely if $\mu$ is a grand canonical Gibbs measure for $(\beta, z)$ with $z_{a} \neq 0 \forall a \in F$, then $\mu$ is canonical and $\mu\left(N^{a}=\infty \forall a \in F\right)>0$.

Lemma 3.6. If $\Phi$ is a uniformly bounded potential, and $\mu$ a canonical Gibbs measure for some $\beta$, such that $\mu\left(N^{a} \geqq 1 \forall a \in F\right)>0$, then $\mu$ is everywhere dense. Conversely if $\mu$ is canonical and everywhere dense, then $\mu\left(N^{a}=\infty \forall a \in F\right)>0$.

Proof. If $\mu$ is canonical and $\mu\left(N^{a} \geqq 1 \forall a \in F\right)>0$, then it follows from [7, Lemma 
5.33] that $\mu\left(N^{a}=\infty \forall a \in F\right)>0$. For the direct part, suppose there is a $\Lambda$ and a $\xi$ in $\Omega_{\Lambda}$ with $\mu_{\Lambda}(\xi)=0$. Since $\mu\left(N^{a}=\infty \forall a \in F\right)>0$, there is a $\Delta \supset \Lambda$ and an $\eta$ in $\Omega_{\Delta}$ with $\mu_{\Delta}(\eta)$ $>0$ and $N_{\Delta}^{a}(\eta) \geqq N_{\Lambda}^{a}(\xi) \forall a \in F$. Select an $\omega$ in $\Omega_{\Delta}$ with $N_{\Delta}(\omega)=N_{\Delta}(\eta)$ and with $\omega_{\Lambda}=\xi$. Then from (2.6), (2.7), it follows that $\mu_{\Delta}(\omega)>0$, and hence $\mu_{\Lambda}(\xi)>0$, which is a contradiction. (Compare Lemma 4.3 in [11].)

Conversely, if $\mu$ is everywhere dense, then trivially $\mu\left(N^{a} \geqq 1 \forall a \in F\right)>0$, and again from [7, Lemma 5.33], it follows that $\mu\left(N^{a}=\infty \forall a \in F\right)>0$.

Proof of Theorem 3.5. The converse part is the easier one. Let $F=\left(a_{1}, \ldots, a_{|F|}\right)$. If $\mu$ is grand canonical Gibbs it is canonical as is seen from (2.4-2.7) or Remark A1 in the appendix. Let $\Lambda_{n}$ denote a sequence of translates of some volume $\Lambda_{0}$ with $\left|\Lambda_{0}\right|=|F|$, and consider $\xi^{0}$ in $\Omega_{\Lambda_{0}}$ with $\left(\xi^{0}\right)=\left(a_{1}, \ldots, a_{|F|}\right)$. Denote by $\xi^{n}$ the translates of $\xi^{0}$ in $\Omega_{\Lambda_{n}}$ and let dist $\left(\Lambda_{0}, \Lambda_{n}\right) \rightarrow \infty$. Since $z_{a} \neq 0 \forall a \in F$ and since the potential is uniformly bounded, it follows from (2.4), (2.5) that

$$
\liminf _{n \rightarrow \infty} \mu_{\Lambda}\left(\xi^{n}\right)>0 .
$$

Hence $\mu\left(N^{a}=\infty \forall a \in F\right)>0$.

For the direct part, we assume that $\mu$ is an extreme canonical Gibbs state for $(\beta, \Phi)$ with the property that

$$
\mu\left(N^{a} \geqq 1 \text { for all } a \text { in } F\right)>0 .
$$

For $N$ in $\mathbb{Z}^{F}$ we denote by $\pi(N)$ the set

$$
\left\{\left(\phi, \phi^{\prime}\right): \exists \text { finite } \Lambda: \phi \in \Omega_{\Lambda}, \phi^{\prime} \in \Omega_{\Lambda}, \phi_{\delta \Lambda}=\phi_{\delta \Lambda}^{\prime} \text {, and } N_{\Lambda}(\phi)-N_{\Lambda}\left(\phi^{\prime}\right)=N\right\} \text {. }
$$

Choose an arbitrary representative $\left(\zeta, \zeta^{\prime}\right)^{\circ}$ of $\pi(N)$ in a volume $\Delta_{0}$ and let $\left\{\Delta_{0}\right\}$ be a sequence of translates of $\Delta_{0}\left(\Delta_{n}=T_{n}\left(\Delta_{0}\right)\right)$ with the properties that $\operatorname{dist}\left(\Delta_{0}, \Delta_{n}\right) \rightarrow \infty$ as $n \rightarrow \infty$ and $\operatorname{dist}\left(\Lambda, \Delta_{n}\right)>2 D$ for all $n$.

Define configurations $\eta^{n}, \eta^{\prime n}$ in $\Delta_{n}$ by:

$$
\eta^{n}=T_{n}\left(\zeta^{\prime}\right) \text { and } \eta^{\prime n}=T_{n}(\zeta)
$$

Then we obtain for all $\left(\xi, \xi^{\prime}\right)$ in $\pi(N)$ :

$$
\begin{aligned}
& \text { i. }\left(\xi, \eta^{n}\right)_{\delta\left(\Lambda \cup \Delta_{n}\right)}=\left(\xi^{\prime}, \eta^{\prime \prime}\right)_{\delta\left(\Lambda \cup \Delta_{n}\right)} \\
& \text { ii. } N_{\Lambda \cup \Delta_{n}}\left(\xi, \eta^{n}\right)=N_{\Lambda}(\xi)+N_{\Delta_{n}}\left(\eta^{n}\right)=N_{\Lambda}(\xi)+N_{\Delta_{0}}\left(\zeta^{\prime}\right) \\
& =N+N_{\Lambda}\left(\xi^{\prime}\right)+N_{\Delta_{0}}(\zeta)-N=N_{\Lambda \cup \Delta_{n}}\left(\xi^{\prime}, \eta^{\prime n}\right) .
\end{aligned}
$$

Hence we obtain from the canonical prescription (2.6) and (2.7) and (3.2),

$$
\mu_{\Lambda \cup \Delta_{n}}\left(\xi, \eta^{n}\right)=\mu_{\Lambda \cup \Delta_{n}}\left(\xi^{\prime}, \eta^{\prime n}\right) \exp \left[-\beta E_{\Lambda \cup \Delta_{n}}\left(\xi, \eta^{n}\right)+\beta E_{\Lambda \cup \Delta_{n}}\left(\xi^{\prime}, \eta^{\prime n}\right)\right] .
$$

But when dist $\left(\Lambda, \Delta_{n}\right)>2 D$, we have

$$
\begin{aligned}
-\beta E_{\Lambda \cup \Delta_{n}}\left(\xi, \eta^{n}\right)+\beta E_{\Lambda \cup \Delta_{n}}\left(\xi^{\prime}, \eta^{\prime n}\right) & =-\beta\left[E_{\Lambda}(\xi)-E_{\Lambda}\left(\xi^{\prime}\right)\right]-\beta\left[E_{\Delta_{n}}\left(\eta^{n}\right)-E_{\Delta_{n}}\left(\eta^{\prime n}\right)\right] \\
& \equiv \log q_{\Lambda}\left(\xi, \xi^{\prime}\right)+\log q_{\Delta_{n}}\left(\eta^{n}, \eta^{\prime n}\right) .
\end{aligned}
$$

As $\mu$ is an extreme canonical Gibbs state, $\mu$ is trivial on $\mathscr{E}_{\infty}[7$, Theorem 1.32] and therefore on $\mathscr{F}_{\infty}$. Thus, from [6]

$$
\lim _{n \rightarrow \infty}\left|\mu_{\Lambda \cup \Delta_{n}}\left(\xi, \eta^{n}\right)-\mu_{\Lambda}(\xi) \mu_{\Delta_{n}}\left(\eta^{n}\right)\right|=0
$$


and

$$
\lim _{n \rightarrow \infty}\left|\mu_{\Lambda \cup \Delta_{n}}\left(\xi^{\prime}, \eta^{\prime n}\right)-\mu_{\Lambda}\left(\xi^{\prime}\right) \mu_{\Delta_{n}}\left(\eta^{\prime n}\right)\right|=0 .
$$

Combining (3.3)-(3.6) and the boundedness of the potential, we obtain

$$
\lim _{n \rightarrow \infty}\left(\mu_{\Lambda}(\xi) \mu_{\Delta_{n}}\left(\eta^{n}\right)-\mu_{\Lambda}\left(\xi^{\prime}\right) \mu_{\Delta_{n}}\left(\eta_{n}^{\prime}\right) q_{\Lambda}\left(\xi, \xi^{\prime}\right) q_{\Delta_{n}}\left(\eta^{n}, \eta^{\prime n}\right)\right)=0 .
$$

Hence, from Lemma 3.6 and again the boundedness of $\Phi$ :

$$
\lim _{n \rightarrow \infty}\left[\frac{\mu_{\Lambda}(\xi)}{\mu_{\Lambda}\left(\xi^{\prime}\right)} q_{\Lambda}\left(\xi, \xi^{\prime}\right)^{-1}-\frac{\mu_{\Delta_{n}}\left(\eta_{n}^{\prime}\right)}{\mu_{\Delta_{n}}\left(\eta_{n}\right)} q_{\Delta_{n}}\left(\eta^{n}, \eta^{\prime n}\right)\right] \mu_{\Delta_{n}}\left(\eta^{n}\right)=0 .
$$

We claim that it is possible to find a subsequence $n_{k}$ and $\eta^{0}$ such that

$$
\lim _{n_{k} \rightarrow \infty} \mu_{\Delta_{n_{k}}}\left(\eta^{n_{k}}\right)>0
$$

This claim follows from an argument, which for reasons of clarity, will be stated in Lemma A.3 of the appendix. (It basically follows the above reasoning.)

From (3.8) we obtain for at least one subsequence $T_{n}$, and for all couples $\left(\xi, \xi^{\prime}\right)$ in $\pi(N)$ :

$$
\frac{\mu_{\Lambda}(\xi)}{\mu_{\Lambda}\left(\xi^{\prime}\right)} q_{\Lambda}\left(\xi, \xi^{\prime}\right)^{-1}=\lim _{n \rightarrow \infty} \frac{\mu_{\Delta_{n}}\left(\eta_{n}^{\prime}\right)}{\mu_{\Delta_{n}}\left(\eta_{n}\right)} q_{\Delta_{n}}\left(\eta^{n}, \eta^{\prime n}\right) .
$$

The limit in (3.9) does not depend on the chosen subsequence, nor on the particular choice of $T_{n}, \eta, \xi, \xi^{\prime}$, provided the configurations belong to $\pi(N)$. It therefore defines a function $g$ on $\mathbb{Z}^{F}$, and it may be checked that $g(N+M)=$ $g(N) g(M)$ and $g(-N)=g(N)^{-1}$. To avoid repetition, see the corresponding argument for the transition microcanonical-canonical in Lemma 4.5. It follows that there is a $z=(z(a))_{a \in F}$ such that

$$
g(N)=\prod_{a \in F}(z(a))^{N^{a}} .
$$

Since $|F| \geqq 2, z$ is uniquely determined. We have therefore obtained that for all $\Lambda$, all $\xi, \xi^{\prime}$ in $\Omega_{\Lambda}$ with $\xi_{\delta \Lambda}=\xi_{\delta \Lambda}^{\prime}$ :

$$
\frac{\mu_{\Lambda}(\xi)}{\mu_{\Lambda}\left(\xi^{\prime}\right)}=\prod_{a \in F}(z(a))^{N_{\Lambda}^{a}(\xi)-N_{\Lambda}^{a}\left(\xi^{\prime}\right)} \exp \left[-\beta E_{\Lambda}(\xi)+\beta E_{\Lambda}\left(\xi^{\prime}\right)\right]
$$

It follows from a compatibility argument (see the corresponding result in the proof of Theorem 3.3) that $\mu$ is a grand canonical Gibbs state for $\beta$ and $z$. $\mu$ is an extreme grand canonical Gibbs state as it is an extreme canonical Gibbs state.

We conclude this section with a number of remarks and counterexamples.

Example 3.7 (Incommensurable potentials). Let $v=1, F=\{-1,+1\}, \alpha, \gamma \in \Omega_{\{0,1\}}$ with $\alpha=(+1,+1), \gamma=(-1,-1)$, and $J_{\alpha} / J_{\gamma} \notin Q .\left(J_{\alpha}, J_{\gamma} \neq 0\right)$, all other coupling constants being zero. Then (2.3) reads: $E_{\Lambda}(\xi \mid \eta)=J_{\alpha} M_{\Lambda}^{\alpha}(\xi, \eta)+J_{\gamma} M_{\Lambda}^{\gamma}(\xi, \eta)$ (with $\left.M_{\Lambda}^{\alpha}()=.N_{\Lambda \cup \partial \Lambda}^{\alpha}()-.N_{\partial \Lambda}^{\alpha}().\right)$. 
Choose $\beta_{\alpha} \neq \beta_{\gamma}$, and define conditional probability distributions $\mu_{\Lambda, \beta}(. \mid$.), with $\beta=\left(\beta_{\alpha}, \beta_{\gamma}\right)$, by:

$$
\mu_{\Lambda, \beta}(\xi \mid \eta)=\left(1 / Z_{\Lambda}(\eta)\right) \exp \left[-\beta_{\alpha} J_{\alpha} M_{\Lambda}^{\alpha}(\xi, \eta)-\beta_{\gamma} J_{\gamma} M_{\Lambda}^{\gamma}(\xi, \eta)\right] .
$$

The family $\mu_{\Lambda, \beta}(. \mid$.$) is consistent. It follows that every limit point of \mu_{\Lambda, \beta}(. \mid$. $)$ has the given $\mu_{\Lambda, \beta}(. \mid$.$) as conditional probability measures with respect to \mathscr{F}_{\Lambda}$. The existence of a limit point $\mu_{\beta}$ is guaranteed by compactness arguments.

Clearly $\mu_{\beta}$ is not a canonical Gibbs measure. (Calculate e.g. $\mu_{\Lambda, \beta}\left(\xi \eta, N_{\Lambda}\right)=$ $\mu_{\Lambda, \beta}\left(\xi, N_{\Lambda} \mid \eta\right) / \mu_{\Lambda, \beta}\left(N_{\Lambda} \mid \eta\right)$.)

On the other hand, $\mu_{\beta}$ is a microcanonical Gibbs measure since the specification of a value for $E_{\Lambda}\left(. \mid\right.$.) uniquely determines, by the incommensurability of $J_{\alpha}$ and $J_{\gamma}$, the numbers $N_{\Lambda}^{\alpha}$ and $N_{\Lambda}^{\nu}$. (Compare [9].)

The example shows that the commensurability of the coupling constants is an essential condition. Of course, it is possible to eliminate pathologies of the kind described in the example by the requirement that the function of the energy difference, which relates probabilities of local configurations with the same particle number should be a continuous function of the energy difference. (cf. Lemma 4.4.) However, it is hard to formulate an elegant a priori condition.

Remark 3.8. Not everywhere dense microcanonical Gibbs measures. Microcanonical Gibbs measures that are not everywhere dense are abundant in the following example. The potential is that of the ferromagnetic Ising chain in zero-external field: $F=\{-1,1\}$ and

$$
E_{\Lambda}(\xi \mid \eta)=-J \sum_{x ;[x, x+1] \cap \Lambda \neq \phi} S_{x} S_{x+1}(\xi, \eta) .
$$

Introduce the following configurations and the point measures associated with them (using the same notation):

$$
\begin{array}{ll}
\delta_{+}:\left(\delta_{+}\right)_{n}=+1 & \forall n, \\
\delta_{-}:\left(\delta_{-}\right)_{n}=-1 & \forall n,
\end{array}
$$

and the families

$$
\begin{aligned}
\left\{\delta_{+-}^{n}\right\}_{n \in \mathbb{Z}^{*}}:\left(\delta_{+-}^{n}\right)_{m} & =+1 & & \text { if } m<n \\
& =-1 & & \text { if } m \geqq n, \\
\left\{\delta_{-+}^{n}\right\}_{n \in \mathbb{Z}^{*}}:\left(\delta^{n}\right)_{m} & =-1 & & \text { if } m<n \\
& =+1 & & \text { if } m \geqq n .
\end{aligned}
$$

Next define the transformation $I^{o}, I^{e}$ on $\Omega$ :

$$
\left(I^{o} \xi\right)_{x}=\xi_{x}(-1)^{i+1} \text { and }\left(I^{e} \xi\right)_{x}=\xi_{x}(-1)^{i}
$$

It may be checked that the measures $\delta_{+}, \delta_{-}, \delta_{+-}^{n}, \delta_{-+}^{n}$, and their transforms under $I^{o}$ and $I^{e}$, are all microcanonical Gibbs measures. For the original measures, the argument goes as follows. The specification of boundary spins, of $N_{\Lambda}^{+}, N_{\Lambda}^{-}$and the value of $E_{\Lambda}$, determines a unique configuration, whence conditions (2.7) and (2.8) easily follow. $\delta_{+}, \delta_{-}, \delta_{+-}^{n}, \delta_{-+}^{n}$ constitute the set of ground states of the Ising model 
and correspond to the $\beta=+\infty$ case in the canonical and grand canonical language.

The same argument works for the transformed measures. These are the ceiling states $(\beta=-\infty)$ for the Ising model. It would be interesting to fully characterize the set of not everywhere dense microcanonical Gibbs states: are there other possibilities besides ground or ceiling states or states where some particle type is excluded?

Remark 3.9. For highly non translation invariant potentials, a microcanonical Gibbs measure need not be canonical Gibbs.

Let $L=\mathbb{N}^{o}, F=\{a, b\}$, and use $I($.$) to denote the characteristic function for the$ event between parentheses. Define an energy of the form

$$
E_{\Lambda}(\xi \mid \eta)=\sum_{i ;[i, i+1] \wedge \Lambda \neq \phi} J_{i} I\left(S_{i}(\xi, \eta)=S_{i+1}(\xi, \eta)=a\right),
$$

where $J_{i}=10^{-i}$. Then the $J_{i}$ are commensurable, yet every energy level is nondegenerate, since

$$
\sum_{i \in M} J_{i}=\sum_{j \in N} J_{j} \quad \text { if and only if } \quad M=N .
$$

Define conditional probability distributions $\mu_{\Lambda, \beta}$ with $\beta \in l^{\infty}\left(\mathbb{N}^{o}\right)$, and such that $\beta_{i} \neq \beta_{j}$ for at least one pair $i \neq j$, by

$$
\mu_{\Lambda, \beta}(\xi \mid \eta)=\left(1 / Z_{\Lambda}(\eta)\right) \exp \left[-\sum_{\substack{i ; \\[,,+1] \cap \Lambda \neq \phi}} \beta_{i} J_{i} I\left(S_{i}(\xi, \eta)=S_{i+1}(\xi, \eta)=a\right)\right] .
$$

By the consistency of the $\mu_{\boldsymbol{A}, \boldsymbol{\beta}}$ and a compactness argument there does exist at least one measure $\mu_{\beta}$ with the given $\mu_{A, \beta}$ as conditional probability measures with respect to $\mathscr{F}_{\Lambda}$. Clearly, $\mu_{\beta}$ is not a canonical Gibbs measure.

On the other hand, $\mu_{\beta}$ is a microcanonical Gibbs measure since the specification of a value of $E_{\Lambda}(. \mid$.) uniquely determines a configuration.

\section{Proof of the Main Result}

For the sake of simplicity in formulation and notation, the results will be formulated and proven in a translation invariant setting. The adaptation to approximately periodic potentials is straightforward: it suffices to choose appropriate "adapted sequences" in the following proofs.

Lemma 4.1. Let $\mu$ be a microcanonical Gibbs measure for a finite range potential. If $\phi$, $\eta \in \Omega_{\Lambda}$ with

i) $N_{\Lambda}(\phi)=N_{\Lambda}(\eta)$,

ii) $\phi_{\delta \Lambda}=\eta_{\delta \Lambda}$,

iii) $E_{\Lambda}(\phi)=E_{\Lambda}(\eta)$

then $\mu\left(S_{\Lambda}=\phi\right) \equiv \mu_{\Lambda}(\phi)=\mu_{\Lambda}(\eta)$

Proof. It follows from (ii) and (iii) that for all $\omega$ in $\Omega$ :

$$
E_{\Lambda}\left(\phi \mid \omega_{\Lambda^{c}}\right)=E_{\Lambda}\left(\eta \mid \omega_{\Lambda^{c}}\right),
$$


and together with (i) and (2.9) this implies $v_{\Lambda}(\phi \mid \omega)=v_{\Lambda}(\eta \mid \omega)$. Hence

$$
\begin{aligned}
\mu_{\Lambda}(\phi) & =\int d \mu\left(\omega_{\Lambda^{c}}, N_{\Lambda}(\omega), E_{\Lambda}\left(\omega_{\Lambda} \mid \omega_{\Lambda^{c}}\right)\right) v_{\Lambda}(\phi \mid \omega) \\
& =\int d \mu\left(\omega_{\Lambda^{c}}, N_{\Lambda}(\omega), E_{\Lambda}\left(\omega_{\Lambda} \mid \omega_{\Lambda^{c}}\right)\right) v_{\Lambda}(\eta \mid \omega) \\
& =\mu_{\Lambda}(\eta) .
\end{aligned}
$$

In the remainder of this article, the microcanonical hypothesis will enter only through the above mentioned result.

The following two lemmas are preparatory for the main Lemmas 4.4 and 4.5.

Lemma 4.2. If $\Phi$ is translation invariant and has finite range, $\Sigma(\Phi)$ is a group.

Proof. Let $\rho_{1}, \rho_{2} \in \Sigma$. Then, $\rho_{i}=\sigma_{i}-\tau_{i}$, with

$$
\begin{aligned}
& \sigma_{i}=E_{\Lambda_{\imath}}\left(\omega^{i}\right), \\
& \tau_{i}=E_{\Lambda_{\imath}}\left(\eta^{i}\right),
\end{aligned}
$$

for some $\Lambda_{1}, \Lambda_{2}$ and $\omega^{i} \in \Omega_{\Lambda_{1}}, \eta^{i} \in \Omega_{\Lambda_{i}}$ with $N_{\Lambda_{i}}\left(\omega^{i}\right)=N_{\Lambda_{i}}\left(\eta^{i}\right)$ and $\left(\omega^{i}\right)_{\delta \Lambda_{\imath}}=\left(\eta^{i}\right)_{\delta \Lambda_{i}}$; $i=1$, 2. (Definition 3.1). Let $T$ be a translation such that dist $\left(\Lambda_{1}, T\left(\Lambda_{2}\right)\right)>2 D$. Then

$$
\begin{aligned}
& E_{\Lambda_{2}}\left(\omega^{2}\right)=E_{T\left(\Lambda_{2}\right)}\left(T \omega^{2}\right), \\
& E_{\Lambda_{2}}\left(\eta^{2}\right)=E_{T\left(\Lambda_{2}\right)}\left(T \eta^{2}\right),
\end{aligned}
$$

and

$$
\begin{aligned}
& E_{\Lambda_{1} \cup T\left(\Lambda_{2}\right)}\left(\omega^{1},\left(T \omega^{2}\right)\right)-E_{\Lambda_{1} \cup T\left(\Lambda_{2}\right)}\left(\eta^{1},\left(T \eta^{2}\right)\right) \\
& \quad=E_{\Lambda_{1}}\left(\omega^{1}\right)+E_{\Lambda_{2}}\left(\omega^{2}\right)-E_{\Lambda_{1}}\left(\eta^{1}\right)-E_{\Lambda_{2}}\left(\eta^{2}\right)=\rho_{1}+\rho_{2} .
\end{aligned}
$$

It follows that $\rho_{1}+\rho_{2} \in \Sigma$ if $\rho_{1}, \rho_{2} \in \Sigma$.

Lemma 4.3. Let $\mu$ be an everywhere dense, extreme microcanonical Gibbs measure for a translation invariant, finite range potential. Let $\left\{\Lambda_{n}\right\}$ denote a sequence of translates of a volume $\Lambda_{0}$ such that dist $\left(\Lambda_{0}, \Lambda_{n}\right) \rightarrow \infty$ as $n \rightarrow \infty$. Let $\zeta^{n}$ denote the corresponding translates in $\Lambda_{n}$ of some configuration $\zeta^{0}$ in $\Omega_{\Lambda_{0}}$. Then $\lim \sup \mu_{\Lambda_{n}}\left(\zeta^{n}\right)>0$.

Proof: Suppose $\lim _{n \rightarrow \infty} \mu_{\Lambda_{n}}\left(\zeta^{n}\right)=0$.

Let $\eta^{n} \in \Omega_{\Lambda_{n}}$ denote the translates of some configuration $\eta^{0}$ in $\Omega_{\Lambda_{0}}$. Define $\Delta_{n}=\Lambda_{n} \cup \partial \Lambda_{n}$. Choose an arbitrary configuration $\phi^{0}$ in $\partial \Lambda_{0}$, and let $\left\{\phi^{n}\right\}$ be its translates in $\partial \Lambda_{n}$. The microcanonical hypothesis implies with Lemma 4.1 and the translation invariance of the potential that, whenever dist $\left(\Lambda_{0}, \Lambda_{n}\right)>2 D$ :

$$
\mu_{\Delta_{0} \cup \Delta_{n}}\left(\eta^{0}, \phi^{0}, \zeta^{n}, \phi^{n}\right)=\mu_{\Delta_{0} \cup \Delta_{n}}\left(\zeta^{0}, \phi^{0}, \eta^{n}, \phi^{n}\right) .
$$

Since $\mu$ is extreme microcanonical, it is trivial on $\mathscr{D}_{\infty}$ (cf. Sect. 2), and therefore on $\mathscr{F}_{\infty}$. Thus we obtain from [6]:

$$
\lim _{n \rightarrow \infty}\left|\mu_{\Delta_{0} \cup \Delta_{n}}\left(\eta^{0}, \phi^{0}, \zeta^{n}, \phi^{n}\right)-\mu_{\Delta_{0}}\left(\eta^{0}, \phi^{0}\right) \mu_{\Delta_{n}}\left(\zeta^{n}, \phi^{n}\right)\right|=0
$$

and

$$
\lim _{n \rightarrow \infty}\left|\mu_{\Delta_{0} \cup \Delta_{n}}\left(\zeta^{0}, \phi^{0}, \eta^{n}, \phi^{n}\right)-\mu_{\Delta_{0}}\left(\zeta^{0}, \phi^{0}\right) \mu_{\Delta_{n}}\left(\eta^{n}, \phi^{n}\right)\right|=0
$$


Then (4.1), (4.2), (4.3) and the hypothesis that $\lim _{n \rightarrow \infty} \mu_{\Lambda_{n}}\left(\zeta^{n}\right)=0$ imply for all $\phi^{0}$ :

$$
\lim _{n \rightarrow \infty} \mu_{\Delta_{0}}\left(\zeta^{0}, \phi^{0}\right) \mu_{\Delta_{n}}\left(\eta^{n}, \phi^{n}\right)=0 .
$$

Since $\mu$ is everywhere dense, we have for all $\phi^{0}$ :

$$
\lim _{n \rightarrow \infty} \mu_{\Delta_{n}}\left(\eta^{n}, \phi^{n}\right)=0
$$

Thus, for all $\eta^{0} \in \Omega_{\Lambda_{0}}: \lim _{n \rightarrow \infty} \mu_{\Delta_{n}}\left(\eta^{n}\right)=0$, which is a contradiction since $\sum_{\eta^{0}} \mu_{\Delta_{n}}\left(\eta^{n}\right)=1$.

Lemma 4.4. Let $\mu$ be an everywhere dense, extreme microcanonical Gibbs measure for a translation invariant, finite range potential. Then there exists a function $f$ on $\Sigma$ such that for all $\Lambda$, and any couple of configurations $\phi, \phi^{\prime}$ in $\Omega_{\Lambda}$ with
i) $N_{\Lambda}(\phi)=N_{\Lambda}\left(\phi^{\prime}\right)$
ii) $\phi_{\delta \Lambda}=\phi_{\delta \Lambda}^{\prime}$,

then

$$
\frac{\mu_{\Lambda}(\phi)}{\mu_{\Lambda}\left(\phi^{\prime}\right)}=f\left(E_{\Lambda}(\phi)-E_{\Lambda}\left(\phi^{\prime}\right)\right)
$$

Proof. Let $\pi(\sigma)$ be the set of couples of configurations $\left(\phi, \phi^{\prime}\right)$ belonging to a given spectral value $\sigma$, i.e. there is a finite $\Lambda$ such that $\phi, \phi^{\prime} \in \Omega_{\Lambda}$ and

i) $N_{\Lambda}(\phi)=N_{\Lambda}\left(\phi^{\prime}\right)$,

ii) $\phi_{\delta \Lambda}=\phi_{\delta \Lambda}^{\prime}$,

iii) $E_{\Lambda}(\phi)-E_{\Lambda}\left(\phi^{\prime}\right)=\sigma$.

Choose an arbitrary representative $\left(\zeta, \zeta^{\prime}\right)$ of $\pi(\sigma)$ in a volume $\Delta_{0}$. Let $\left\{\Delta_{n}\right\}$ be a sequence of translates of $\Delta_{0}\left(\Delta_{n}=T_{n} \Delta_{0}\right)$ such that

$$
\operatorname{dist}\left(\Delta_{0}, \Delta_{n}\right) \rightarrow \infty \text { and dist }\left(\Lambda, \Delta_{n}\right)>2 D \text { for all } n \text {. }
$$

Define configurations $\eta^{n}, \eta^{\prime n}$ in $\Delta_{n}$ by

$$
\begin{aligned}
\eta^{n} & =T_{n}\left(\zeta^{\prime}\right), \\
\eta^{\prime n} & =T_{n}(\zeta) .
\end{aligned}
$$

Then for all $\left(\phi, \phi^{\prime}\right)$ in $\pi(\sigma)$ :

a) $N_{\Lambda \cup \Delta_{n}}\left(\phi, \eta^{n}\right)=N_{\Lambda \cup \Delta_{n}}\left(\phi^{\prime}, \eta^{\prime n}\right)$ (from (i), (4.5), (4.6))

b) $\left(\phi, \eta^{n}\right)_{\delta\left(\Delta \cup \Delta_{n}\right)}=\left(\phi^{\prime}, \eta^{\prime n}\right)_{\delta\left(\Delta \cup \Delta_{n}\right)}$ from (ii), and (4.5), (4.6)

c) $E_{\Lambda \cup \Delta_{n}}\left(\phi, \eta^{n}\right)=E_{\Lambda}(\phi)+E_{\Delta_{n}}\left(\eta^{n}\right)=E_{\Lambda}(\phi)+E_{\Delta_{0}}\left(\zeta^{\prime}\right)$

$$
=E_{\Lambda}\left(\phi^{\prime}\right)+\sigma+E_{\Delta_{0}}(\zeta)-\sigma=E_{\Lambda \cup \Delta_{n}}\left(\phi^{\prime}, \eta^{\prime n}\right)
$$

from (4.4), (4.5), (4.6) and the translation invariance. 
Properties (a), (b), (c) and Lemma 4.1 imply:

$$
\mu_{\Lambda \cup \Delta_{n}}\left(\phi, \eta^{n}\right)=\mu_{A \cup \Delta_{n}}\left(\phi^{\prime}, \eta^{\prime n}\right) .
$$

Since $\mu$ is an extreme microcanonical Gibbs measure, $\mu$ is trivial on $\mathscr{D}_{\infty}$ and therefore on $\mathscr{F}_{\infty}$. But then it follows from [6] that

$$
\begin{gathered}
\lim _{n \rightarrow \infty}\left|\mu_{\Lambda \cup \Delta_{n}}\left(\phi, \eta^{n}\right)-\mu_{\Lambda}(\phi) \mu_{\Delta_{n}}\left(\eta^{n}\right)\right|=0, \\
\lim _{n \rightarrow \infty}\left|\mu_{\Lambda \cup \Delta_{n}}\left(\phi^{\prime}, \eta^{\prime n}\right)-\mu_{\Lambda}\left(\phi^{\prime}\right) \mu_{\Delta_{n}}\left(\eta^{\prime n}\right)\right|=0 .
\end{gathered}
$$

Then (4.7), (4.8), (4.9), the everywhere density of $\mu$ and Lemma 4.3 imply

$$
\frac{\mu_{\Lambda}(\phi)}{\mu_{\Lambda}\left(\phi^{\prime}\right)}=\frac{\limsup _{n \rightarrow \infty} \mu_{\Delta_{n}}\left(\eta^{\prime n}\right)}{\limsup _{n \rightarrow \infty} \mu_{\Delta_{n}}\left(\eta^{n}\right)} .
$$

The quotient of the limits clearly only depends on $\sigma=E_{\Lambda}(\phi)-E_{\Lambda}\left(\phi^{\prime}\right)$, and hence defines a function on $\Sigma$.

Lemma 4.5. Assume the conditions of Lemma 4.4 to hold. The function $f$ on $\Sigma$ defined in Lemma 4.4. satisfies:

$$
\begin{aligned}
& f(\sigma+\tau)=f(\sigma) f(\tau), \\
& f(-\sigma)=f(\sigma)^{-1} \text { for all } \sigma, \tau \text { in } \Sigma .
\end{aligned}
$$

If in addition the potential satisfies the commensurability condition, there exists a $\beta$ in $\mathbb{R}$ such that $f(\sigma)=\exp [-\beta \sigma]$ for all $\sigma$ in $\Sigma$. If $\Sigma \neq\{0\}, \beta$ is uniquely determined. Proof. To prove the first statement, suppose the couple $\left(\phi, \phi^{\prime}\right)$ belongs to $\pi(\sigma)$, and the couple $\left(\eta, \eta^{\prime}\right)$ belongs to $\pi(\tau)$. We may assume $\phi$ and $\phi^{\prime} \in \Omega_{\Lambda}, \eta$ and $\eta^{\prime} \in \Omega_{\Delta}$ with dist $(\Lambda, \Delta)>2 D$. Then

$$
\sigma=E_{\Lambda}(\phi)-E_{\Lambda}\left(\phi^{\prime}\right)+E_{\Delta}(\eta)-E_{\Delta}(\eta)=E_{\Delta \cup \Delta}(\phi, \eta)-E_{\Lambda \cup \Delta}\left(\phi^{\prime}, \eta\right),
$$

and similarly

$$
\tau=E_{\Lambda \cup \Delta}\left(\phi^{\prime}, \eta\right)-E_{\Lambda \cup \Delta}\left(\phi^{\prime}, \eta^{\prime}\right) .
$$

Hence

$$
\begin{aligned}
f(\sigma) f(\tau) & =\frac{\mu_{\Lambda \cup \Delta}(\phi, \eta)}{\mu_{\Lambda \cup \Delta}\left(\phi^{\prime}, \eta\right)} \cdot \frac{\mu_{\Lambda \cup \Delta}\left(\phi^{\prime}, \eta\right)}{\mu_{\Lambda \cup \Delta}\left(\phi^{\prime}, \eta^{\prime}\right)} \\
& =\frac{\mu_{\Delta \cup \Delta}(\phi, \eta)}{\mu_{\Lambda \cup \Delta}\left(\phi^{\prime}, \eta^{\prime}\right)}=f(\sigma+\tau),
\end{aligned}
$$

since $\sigma+\tau=E_{A \cup \Delta}(\phi, \eta)-E_{A \cup \Delta}\left(\phi^{\prime}, \eta^{\prime}\right)$ and

i) $N_{\Lambda \cup \Delta}(\phi, \eta)=N_{\Lambda \cup \Delta}\left(\phi^{\prime}, \eta\right)=N_{\Delta \cup \Delta}\left(\phi^{\prime}, \eta^{\prime}\right)$,

ii) $(\phi, \eta)_{\delta(\Delta \cup \Delta)}=\left(\phi^{\prime}, \eta\right)_{\delta(\Lambda \cup \Delta)}=\left(\phi^{\prime}, \eta^{\prime}\right)_{\delta(\Delta \cup \Delta)}$.

That $f(-\sigma)=f(\sigma)^{-1}$ is immediate. 
Next, assume the commensurability property. If $\Sigma=\{0\}$, the existence of a $\beta$ is trivial. If not, choose $\sigma \neq 0$ in $\Sigma$ and let $f(\sigma)=x$. For any $\tau$ in $\Sigma$ there exist numbers $p$, $q$ in $\mathbb{Z}$ such that $p \sigma=q \tau$. The result then follows from (4.11): $f(\tau)=x^{p / q}$.

Proof of Theorem 3.3. If $\Sigma=\{0\}$, the result is trivial, but $\beta$ is not unique. Let $\Sigma \neq\{0\}$. Lemmas 4.4 and 4.5 have shown that there is a $\beta$ in $\mathbb{R}$ such that

$$
\frac{\mu_{\Lambda}(\phi)}{\mu_{\Lambda}\left(\phi^{\prime}\right)}=\exp \left[-\beta E_{\Lambda}(\phi)+\beta E_{\Lambda}\left(\phi^{\prime}\right)\right]
$$

for all $\Lambda$, all $\phi, \phi^{\prime}$ in $\Omega_{\Lambda}$ with

(i) $N_{\Lambda}(\phi)=N_{\Lambda}\left(\phi^{\prime}\right)$,

(ii) $\phi_{\delta \Lambda}=\phi_{\delta \Lambda}^{\prime}$.

Let $M=\Lambda \cup \partial \Lambda$, and suppose $\omega, \omega^{\prime}$ in $\Omega_{\Lambda}$ satisfy

$$
N_{\Lambda}(\omega)=N_{\Lambda}\left(\omega^{\prime}\right)
$$

Then, from a compatibility argument

$$
\mu_{\Lambda}(\omega)=\sum_{\eta \in \Omega_{\partial \Lambda}} \mu_{M}(\omega, \eta)
$$

which equals, by (4.12):

$$
\begin{aligned}
& =\sum_{\eta \in \Omega_{\partial \Lambda}} \mu_{M}\left(\omega^{\prime}, \eta\right) \exp \left[-\beta\left(E_{M}(\omega \mid \eta)-E_{M}\left(\omega^{\prime} \mid \eta\right)\right)\right] \\
& =\mu\left[I_{\omega^{\prime}} \exp \left[-\beta\left(E_{\Lambda}(\omega \mid .)-E_{\Lambda}\left(\omega^{\prime} \mid .\right)\right)\right]\right] .
\end{aligned}
$$

But (4.14), together with (4.13), is an algebraic version of the canonical DLR Eqs. (2.6), (2.7) ([7, Proposition 2.19]; see also Remark A2 in the appendix below). $\mu$ is necessarily an extreme canonical Gibbs measure, for otherwise it would not be extreme microcanonical.

Since $\mu$ is everywhere dense, it follows in particular that $\mu\left(N^{a} \geqq 1 \forall a \in F\right)>0$, and hence (Lemma 3.6) that $\mu\left(N^{a}=\infty \forall a \in F\right)>0$. Conversely, if $\mu$ is a canonical Gibbs state, it is a microcanonical Gibbs state, and if $\mu\left(N^{a} \geqq 1 \forall a \in F\right)>0$, then $\mu$ is everywhere dense (Lemma 3.6).

\section{Comments}

Apart from the condition of the everywhere density of the measure, the content of Theorem 3.3 is quite clear. That condition was necessary in order to obtain canonical Gibbs states at finite inverse temperatures or grand canonical Gibbs states at finite $\beta$ and nonzero activities $z_{a}$. Yet it is interesting to further clarify that hypothesis.

If the system is one dimensional and the potential is translation invariant (it need not have the commensurability property), it is possible to prove the following result:

Theorem 5.1. Let $\mu$ be a microcanonical Gibbs measure for a translation invariant, finite range potential $\Phi$ in one dimension. Then $\mu$ is everywhere dense if and only if it has infinitely many basic configurations with respect to $\Phi$.

In this connection we have: 
Definition 5.2. Let $\Phi$ be a translation invariant potential of range $D$ on a one dimensional lattice. A measure $\mu$ on $\Omega=F^{\mathbb{Z}}$ has infinitely many basic configurations with respect to $\Phi$ if it is possible to partition the chain into translates $\Lambda_{n}=T_{n}\left(\Lambda_{0}\right)$ of some interval $\Lambda_{0}$ of length $(D-1)$, with $n \in(D-1) \mathbb{Z}$ such that

$$
\mu\left(N^{\xi \eta}(.)=\infty \forall \xi, \eta \in \Omega_{\Lambda_{0}}\right)>0 .
$$

Here $N^{\xi \eta}(\phi)$, for $\phi \in \Omega$, denotes the cardinality of the set

$$
\begin{aligned}
& \left\{n \in(D-1) \mathbb{Z} \mid T_{n}\left(\Lambda_{0}\right)=\Lambda_{n} ; T_{n+1}\left(\Lambda_{0}\right)=\Lambda_{n+1} ;\right. \\
& \left.T_{n}(\xi)=\phi_{\Lambda_{n}} \quad \text { and } \quad T_{n+1}(\eta)=\phi_{\Lambda_{n+1}}\right\} .
\end{aligned}
$$

Thus, the chain is partitioned in cells of length $D-1$, and a basic configuration is a configuration in a set consisting of two nearest neighbour cells.

The reader will remark that Theorem 5.1 is an analogue of Lemma 3.6, but it must be stressed that the occurrence of one basic configuration of a given type does not imply the occurrence of infinitely many of these (cf. Remark 3.8).

Theorem 5.1 will be proven in a separate publication.

We conclude with some open problems.

It would be interesting to extend, if possible, this analysis to the following potentials:

1. long range potentials.

2. not-approximately-periodic potentials. In view of Remark 3.7 it is important to determine the border case for which the equivalence microcanonicalcanonical still holds.

3. incommensurable potentials. Here the problem is presumably not too hard. The first part of Lemma 4.5 holds and it therefore suffices to impose additional conditions guaranteeing the function $f$ obtained there, to be a continuous function of the energy difference, since $\Sigma(\Phi)$ is dense in $\mathbb{R}$.

And finally:

4. Is it possible to characterize the set of not everywhere dense microcanonical Gibbs measures; are there other possibilities besides ground states, ceiling states or states where some particle type is excluded? (corresponding to the cases $\beta=+\infty, \beta=-\infty$, and $\prod_{a \in F} z_{a}=0$, respectively in the grandcanonical setting). For an abstract characterization, see [10].

\section{Appendix}

Remark A.1:Disordered Systems. The purpose of this remark is to show that certain disordered systems do obey our condition of approximate periodicity. To avoid notational complexity, we present a model where the disorder is on the nearest neighbour coupling constants only, but it will be clear from the discussion that this is not restrictive. To simplify even further, we assume that there are no other interactions.

Let $\Omega=\{-1,+1\}$ and $B=\{b \mid b=\{i, j\} \subset L$ with $\operatorname{dist}(i, j)=1\}$ be the set of bonds. Put $B_{\Lambda}=\{b \in B ; b \subset \Lambda\}$. Suppose the bonds take values in some finite set $K$; (In spin glass models $K=\{-J,+J\}$ and in dilute ferromagnets $K=\{0, J\}$ for some 
positive $J$.) If $\mathscr{J}=K^{B}$, and $\mathscr{J}_{\Lambda}=K^{B} \Lambda$, an element $j=\left(j_{b}\right)_{b \in B}$, defines a potential $\Phi^{j}$ in the following way. For $b$ in $B$ and $\xi$ in $\Omega_{b}$ :

$$
\begin{aligned}
& \Phi_{b}^{j}(\xi)=-j_{b} \text { if } \xi=(1,1) \text { or }(-1,-1), \\
& \Phi_{b}^{j}(\xi)=j_{b} \text { if } \xi=(1,-1) \text { or }(-1,1),
\end{aligned}
$$

and $\Phi_{\Lambda}^{j}=0$ whenever $\Lambda \notin B$. Then the local energies read:

$$
E_{\Lambda}^{j}(\xi \mid \eta)=-\sum_{i ;\{i, i+1\} \cap \Lambda \neq \phi} j_{\{i, i+1\}}\left(S_{i} S_{i+1}\right)(\xi, \eta) .
$$

Let us denote $\left(j_{b}\right)_{b \subset \Lambda}$ by $j_{\Lambda}$, and define random variables on $\mathscr{J}$ by:

$$
J_{\Lambda}(j)=j_{\Lambda} \text {. }
$$

The disorder is introduced via a probability measure $p$ on $K$ and the corresponding homogeneous product measure $P$ on $\mathscr{J}$ :

$$
P=\underset{b \in B}{\otimes} p
$$

We shall now show that $P$ almost surely, $\Phi^{()}$is approximately periodic.

If $\Lambda$ is an arbitrary finite set in the lattice, let $\Omega_{\Lambda}^{\infty}$ denote the event that there is an adapted sequence for $\Lambda$ (Definition 2.1 ). Then

$P\left(\Omega_{\Lambda}^{\infty}\right) \geqq P\left(\right.$ There is a sequence of translates $\Lambda_{n}$ of $\Lambda$ such that:

$\operatorname{dist}\left(\Lambda, \Lambda_{n}\right) \rightarrow \infty$ as $n \rightarrow \infty$ and $J_{\bar{X}}=J_{\bar{X}_{n}}$.)

Let $\Lambda_{n}$ be any sequence of $\Lambda$-translates with $\operatorname{dist}\left(\Lambda, \Lambda_{n}\right) \rightarrow \infty$; then the latter probability has the following lower bound:

$$
\begin{aligned}
& \lim _{n \rightarrow \infty} \lim _{k \rightarrow \infty} P\left(\exists m: n \leqq m \leqq k: J_{\bar{X}}=J_{\bar{\lambda}_{m}}\right) \\
& \lim _{n \rightarrow \infty} \lim _{k \rightarrow \infty}\left(1-\sum_{j_{\bar{X}} \in J_{\bar{X}}}\left[p\left(j_{\bar{X}}\right)\left(1-p\left(j_{\bar{X}}\right)\right)^{k-n+1}\right]\right)=1 .
\end{aligned}
$$

Therefore, we have for all finite $\Lambda: P\left(\Omega_{\Lambda}^{\infty}\right)=1$, and the final result follows upon taking the intersection over all finite $\Lambda$ in $L$.

Remark A.2. There exists an interesting and convenient formulation, in algebraic terms, of the theory of equilibrium states. On $\Omega$ a number of transformation groups are introduced.

$\mathscr{U}$ is the group of invertible, local transformations on $\Omega$. (A transformation $U$ is said to be local if there exists a finite $\Lambda$ such that $(U \omega)_{i}=\omega_{i} \forall \omega \in \Omega, \forall i \in \Lambda^{c} . U$ is said to act in $\Lambda$.)

$\mathscr{V}$ is the group of invertible, local transformations, preserving the particle numbers. (That is, if $V$ acts in $\Lambda$, then $N_{\Lambda}(V \omega)=N_{\Lambda}(\omega) \forall \omega \in \Omega$.)

For a potential $\Phi, \mathscr{W}(\Phi)$ is the group of invertible, local transformations, preserving the particle numbers and the conditional energies. (That is, if $W$ acts in $\Lambda$, then $E_{\Lambda}(W \omega \mid \eta)=E_{\Lambda}(\omega \mid \eta) \forall \omega \in \Omega_{\Lambda} \forall \eta \in \Omega_{\Lambda^{c}}$.) Define the following objects: for $U \in \mathscr{U}$, let

$$
U H-H=\lim _{\Lambda \uparrow \mathbb{Z}^{v}}\left(E_{\Lambda} \circ U-E_{\Lambda}\right), \quad U N-N=\lim _{\Lambda \uparrow \mathbb{Z}^{v}}\left(N_{\Lambda} \circ U-N_{\Lambda}\right) .
$$


It may then be shown that, writing $W f=f \circ W$ etc., the following results hold.

i) A state $\mu$ is a microcanonical Gibbs state for the potential $\Phi$ iff $\forall W \in \mathscr{W}(\Phi)$, $\forall f \in C(\Omega), \mu(W f)=\mu(f)$.

ii) A state $\mu$ is a canonical Gibbs state for the potential $\Phi$ at an inverse temperature $\beta$ iff $\forall V \in \mathscr{V}, \forall f \in C(\Omega)$ :

$$
\mu\left(V^{-1} f\right)=\mu(f \exp (-\beta(V H-H))) .
$$

iii) A state $\mu$ is a grand canonical Gibbs state for the potential $\Phi$ at an inverse temperature $\beta$ and a chemical potential $\mu=\left(\mu^{a}\right)_{a}$ iff $\forall U \in \mathscr{U}, \forall f \in C(\Omega)$ :

$$
\mu\left(U^{-1} f\right)=\mu(f \exp (-\beta(U H-H)) \exp (\beta \mu(U N-N))) .
$$

Lemma A.3. Let $\mu$ be an everywhere dense extreme canonical Gibbs measure for a uniformly bounded, finite range potential $\Phi$ at some inverse temperature $\beta$. Let $\left\{\Lambda_{n}\right\}$ denote a sequence of translates of a volume $\Lambda_{0}$ such that dist $\left(\Lambda_{0}, \Lambda_{n}\right) \rightarrow \infty$ as $n \rightarrow \infty$. Let $\zeta^{n}$ denote the corresponding translates in $\Lambda_{n}$ of some configuration $\zeta^{0}$ in $\Omega_{\Lambda_{0}}$. Then

$$
\limsup _{n \rightarrow \infty} \mu_{\Lambda_{n}}\left(\zeta^{n}\right)>0 .
$$

Proof. Suppose

$$
\lim _{n \rightarrow \infty} \mu_{\Lambda_{n}}\left(\zeta^{n}\right)=0 .
$$

Let $\eta^{n} \in \Omega_{\Lambda_{n}}$ denote the translates of some configuration $\eta^{0}$ in $\Omega_{\Lambda_{0}}$. Define $\Delta_{n}=$ $\Lambda_{n} \cup \partial \Lambda_{n}$. Choose an arbitrary configuration $\phi^{0}$ in $\partial \Lambda_{0}$, and let $\left\{\phi^{n}\right\}$ be its translates in $\partial \Lambda_{n}$. The canonical hypothesis implies whenever $\operatorname{dist}\left(\Delta_{0}, \Delta_{n}\right)>2 D$ :

$$
\begin{aligned}
\mu_{\Delta_{0} \cup \Delta_{n}}\left(\eta^{0}, \phi^{0}, \zeta^{n}, \phi^{n}\right)= & \mu_{\Delta_{0} \cup \Delta_{n}}\left(\zeta^{0}, \phi^{0}, \eta^{n}, \phi^{n}\right) \cdot \exp \left[-\beta E_{\Delta_{0} \cup \Delta_{n}}\left(\eta^{0}, \phi^{0}, \zeta^{n}, \phi^{n}\right)\right] . \\
& \cdot \exp \left[\beta E_{\Delta_{0} \cup \Delta_{n}}\left(\zeta^{0}, \phi^{0}, \eta^{n}, \phi^{n}\right)\right] .
\end{aligned}
$$

An easy proof of this statement follows from Remark A.2 in the appendix. (Remark that $N_{\Delta_{0} \cup \Delta_{n}}\left(\eta^{0} \phi^{0}, \zeta^{n} \phi^{n}\right)=N_{\Delta_{0} \cup \Delta_{n}}\left(\zeta^{0} \phi^{0}, \eta^{n} \phi^{n}\right.$.) But when dist $\left(\Delta_{0}, \Delta_{n}\right)>2 D$, we have

$$
\begin{aligned}
& -\beta E_{\Delta \cup \Delta_{n}}\left(\eta^{0}, \phi^{0}, \zeta^{n}, \phi^{n}\right)+\beta E_{\Delta_{0} \cup \Delta_{n}}\left(\zeta^{0}, \phi^{0}, \eta^{n}, \phi^{n}\right) \\
& \quad=-\beta\left[E_{\Delta_{0}}\left(\eta^{0}, \phi^{0}\right)-E_{\Delta_{0}}\left(\zeta^{0}, \phi^{0}\right)\right]-\beta\left[E_{\Delta_{n}}\left(\zeta^{n}, \phi^{n}\right)-E_{\Delta_{n}}\left(\eta^{n}, \phi^{n}\right)\right] \\
& \quad \equiv \log q_{\Delta_{0}}\left(\eta^{0} \phi^{0}, \zeta^{0} \phi^{0}\right)+\log q_{\Delta_{n}}\left(\zeta^{n} \phi^{n}, \eta^{n} \phi^{n}\right) .
\end{aligned}
$$

Since $\mu$ is extreme canonical, it is trivial on $\mathscr{E}_{\infty}[7$, Theorem 1.32] and therefore on $\mathscr{F}_{\infty}$. Thus from [6]:

$$
\lim _{n \rightarrow \infty}\left|\mu_{\Delta_{0} \cup \Delta_{n}}\left(\eta^{0}, \phi^{0}, \zeta^{n}, \phi^{n}\right)-\mu_{\Delta_{0}}\left(\eta^{0}, \phi^{0}\right) \mu_{\Delta_{n}}\left(\zeta^{n}, \phi^{n}\right)\right|=0,
$$

and

$$
\lim _{n \rightarrow \infty}\left|\mu_{\Delta_{0} \cup \Delta_{n}}\left(\zeta^{0}, \phi^{0}, \eta^{n}, \phi^{n}\right)-\mu_{\Delta_{0}}\left(\zeta^{0}, \phi^{0}\right) \mu_{\Delta_{n}}\left(\eta^{n}, \phi^{n}\right)\right|=0
$$

Since $\sup _{n}\left|q_{\Delta_{n}}\left(\zeta^{n} \phi^{n}, \eta^{n} \phi^{n}\right)\right|<\infty$ by the boundedness of $\Phi$, one obtains from (A,2) 
$(\mathrm{A}, 3),(\mathrm{A}, 4)$ and $(\mathrm{A}, 5)$ :

$$
\begin{aligned}
& \lim _{n \rightarrow \infty}\left[\mu_{\Delta_{0}}\left(\eta^{0}, \phi^{0}\right) \mu_{\Delta_{n}}\left(\zeta^{n}, \phi^{n}\right)-\mu_{\Delta_{0}}\left(\zeta^{0}, \phi^{0}\right) \mu_{\Delta_{n}}\left(\eta^{n}, \phi^{n}\right)\right. \\
& \left.\cdot q_{\Delta_{0}}\left(\eta^{0} \phi^{0}, \zeta^{0} \phi^{0}\right) q_{\Delta_{n}}\left(\zeta^{n} \phi^{n}, \eta^{n} \phi^{n}\right)\right]=0 .
\end{aligned}
$$

Now, $(A, 6)$ and $(A, 1)$ imply:

$$
\lim _{n \rightarrow \infty} \mu_{\Delta_{0}}\left(\zeta^{n}, \phi^{0}\right) \mu_{\Delta_{n}}\left(\eta^{n}, \phi^{n}\right) q_{\Delta_{0}}\left(\eta^{0} \phi^{0}, \zeta^{0} \phi^{0}\right) \cdot q_{\Delta_{n}}\left(\zeta^{n} \phi^{n}, \eta^{n} \phi^{n}\right)=0 .
$$

Therefore, since $\mu$ is everywhere dense and $\Phi$ is uniformly bounded:

$$
\lim _{n \rightarrow \infty} \mu_{\Delta_{n}}\left(\eta^{n}, \phi^{n}\right)=0
$$

for all $\eta^{0}$ in $\Omega_{\Lambda_{0}}$, and all $\phi^{0}$ in $\Omega_{\partial \Lambda_{0}}$. But this is a contradiction, since $\forall n$ :

$$
\sum_{\left(\eta^{0}, \phi^{0}\right) \in \Omega_{\Delta_{0}}} \mu_{\Delta_{n}}\left(\eta^{n}, \phi^{n}\right)=1 .
$$

Acknowledgements This research was carried out while the author was a senior research assistant of the N F.W O (National Fund for Scientific Research (Belgium)) It would not have been realised without a previous collaboration with $\mathrm{W} G$ Sullivan Valuable comments by $\mathbf{J}$ De Cannière, $\mathrm{Ph}$ de Smedt, $\mathrm{M}$ Fannes, $\mathrm{H}$ Roos and G Stragier have led to various improvements

\section{References}

1. Baxter, R J : Exactly solved models in statistical mechanics, London: Academic Press 1982

2 Pirogov, S A, Sinai, Ya G : Phase diagrams of classical lattice systems I, II, Theor Math Phys 25, 358-369 (1975) and 26, 61-76 (1976)

3 Sinai, Ya.G : Theory of phase transitions: rigorous results, Oxford: Pergamon Press 1982

4. Bricmont, J, Kuroda, K, Lebowitz, J L : Surface tension and phase coexistence for general lattice systems, preprint (1983)

5. Dobrushin, R L : Description of a random field by means of its conditional probabilities and conditions of its regularity, Theory Probab. Appl 13, 197-224 (1968)

6 Lanford, O E., Ruelle, D.: Observables at infinity and states with short range correlations in statistical mechanics, Commun Math Phys 13, 194-215 (1969)

7. Georgii, H. O.: Canonical Gibbs measures Lecture Notes in Mathematics, Vol. 760 Berlin, Heidelberg, New-York: Springer, 1979

8 Thompson, R L : Equilibrium states in thin energy shells, Mem Am Math Soc 150 (1974)

9. Aizenman, M, Goldstein, S, Lebowitz, J L : Conditional equilibrium and the equivalence of microcanonical and grand canonical ensembles in the thermodynamic limit. Commun. Math. Phys 62, 279-302 (1978)

10 Preston, C.: Canonical and microcanonical Gibbs states, Z. Wahrscheinlichkeitstheorie Verw Geb 46, 125-158 (1979)

11 Sullivan, W, Vanheuverzwijn, P.: On the canonical Gibbs states associated with certain Markov chains, Z Wahrscheinlichkeitstheorie Verw Geb 62, 171-183 (1983)

12 Preston, $\mathrm{C}:$ Random fields, Lecture Notes in Mathematics, Vol 534 Berlin, Heidelberg, New York: Springer, 1976

Communicated by J. Fröhlich

Received October 15, 1983; in revised from February 20, 1985 